\title{
Criteria for Assessing and Recommending Digital Diabetes Tools: A Delphi Study
}

\author{
Dillys LARBI ${ }^{\mathrm{a}}$, Pietro RANDINE ${ }^{\mathrm{a}, \mathrm{b}}$, Eirik ÅRAND ${ }^{\mathrm{a}, \mathrm{b}}$, Meghan BRADWAY $^{\mathrm{a}, \mathrm{c}}$, \\ Konstantinos ANTYPAS ${ }^{\mathrm{a}, \mathrm{d}}$ and Elia GABARRON ${ }^{\mathrm{a}}$ \\ ${ }^{a}$ Norwegian Centre for E-health Research, University Hospital North Norway, Tromsø, \\ Norway \\ ${ }^{\mathrm{b}}$ Department of Computer Science, UiT The Arctic University of Norway, Tromsø, \\ Norway \\ ${ }^{c}$ Department of Clinical Medicine, UiT The Arctic University of Norway, Tromsø, \\ Norway \\ d SINTEF Digital, Oslo, Norway
}

\begin{abstract}
Diabetes self-management, an integral part of diabetes care, can be improved with the help of digital self-management tools such as apps, sensors, websites, and social media. The study objective was to reach a consensus on the criteria required to assess and recommend digital diabetes self-management tools targeting those with diabetes in Norway. Healthcare professionals working with diabetes care from all health regions in Norway were recruited to participate in a three-round Delphi study. In all rounds, the panellists rated criteria identified in a systematic review and interviews on a scale from $0-10$, with the option to provide comments. On a scale of 0:not important to 10:extremely important, the highest rated criteria for assessing and recommending digital diabetes self-management tools were "Usability" and "Information quality", respectively. For assessing apps, "Security and privacy" was one of the lowest rated criteria. Having access to a list of criteria for assessing and recommending digital self-management tools can help diabetes care stakeholders to make informed choices in recommending and choosing suitable apps, websites, and social media for self-management. Future work on quality assessment of digital health tools should place emphasis on security and privacy compliance, to enable diabetes care stakeholders focus on other relevant criteria to recommend or choose and use such tools.
\end{abstract}

Keywords. Diabetes; Mobile Applications; Internet-Based intervention; Social Media; Delphi Technique

\section{Introduction}

Diabetes self-management, often looked upon as a daunting task, is an integral part of diabetes care $[1,2]$. The use of digital self-management tools such as apps, sensors, websites, and social media has been associated with the effectiveness and improved health of individuals with diabetes $[3,4]$. However, some digital self-management tools could also be ineffective or detrimental to users' health. For example, the many in-

1 Corresponding Author: Dillys Larbi, Norwegian Centre for E-health Research, University Hospital of North Norway, Sykehusveien 239019 Tromsø, Norway; E-mail: dillys.larbi@ehealthresearch.no. 
accurate apps for calculating insulin doses could in the worst case, be life-threatening [5]. Therefore, criteria are needed to assist with evaluating and validating these tools.

However, there is little to no information about criteria for quality assessment of digital self-management tools. Individuals with diabetes and their healthcare providers should be given the opportunity to use a list of criteria to assess apps, sensors, websites, and social media before they choose to use and/or recommend them. A Delphi study is an appropriate method for obtaining academic and experience-based consensus on identified evaluation criteria, as it elicits individualized but group-generated information and allows for a wide geographical access to experts [6].

The study objective was to reach a consensus on the criteria required to assess and recommend digital diabetes self-management tools (apps, websites, and social media) targeting the Norwegian diabetes population.

\section{Methods}

Healthcare professionals working within diabetes care from the four health regions in Norway were recruited to participate as panellists in a three-round Delphi study over six weeks (May to June 2020).

From a previous systematic review [7], Usability, Clinical impact, Cognitive impact, Behavioral impact, Feasibility, Engagement, Acceptability and acceptance, and Security and privacy were identified as the 8 criteria categories [7]. In addition, the following criteria were identified from interviews [8] with stakeholder representatives (individuals with diabetes, healthcare professionals, informal caregivers, health authorities, health researchers and developers): Usability, Information quality, Data accessibility, Tailorability, Visual presentation, Remote monitoring, and Automated data recording.

In the first round, panellists rated these criteria on a scale from 0 - not important to 10 - extremely important, with the option to provide comments. Their ratings and comments were analysed and used for the second round. The criteria were then presented together with the average rating and each participant's previous value for each criterion. The third round provided the panellists with another opportunity to change their opinion.

The analysis of the participants' data was done using SPSS version 25. NVivo 12 Pro was used to organize and perform an inductive thematic analysis of the qualitative data. The treatment of personal information in this study was approved by the Data Protection Officer at the University Hospital of North Norway (ref. 2018/3325).

\section{Results}

Fifteen healthcare professionals with an average of 19 years' diabetes care experience were enrolled: $20 \%(3 / 15)$ were medical doctors, $67 \%$ (10/15) were diabetes nurses and the remaining two were a clinical dietitian and an occupational therapist. Females made up $73 \%(11 / 15)$ of the panellists. The average level of experience and observed use of digital diabetes tools, on a scale from 0 - very low to 10 - very high, in the panellists' daily practices were 6.8 and 6.5 , respectively.

With regards to the criteria for recommending digital self-management tools: Information quality had the highest average rating for apps (9.7), websites (9.6), and social media (8.8). The second highest rated criteria, with an average rating of 8.9 , were Usability and Automated data recording for apps. Clinical impact for both websites and 
social media, with an average rating of 8.1 and 7.9 , respectively. With an average rating of 7.2, Usability, Feasibility and Acceptability were the third highest rated criteria for social media, Usability (7.9) for websites and Clinical impact (8.8) for apps, see Figure 1 .

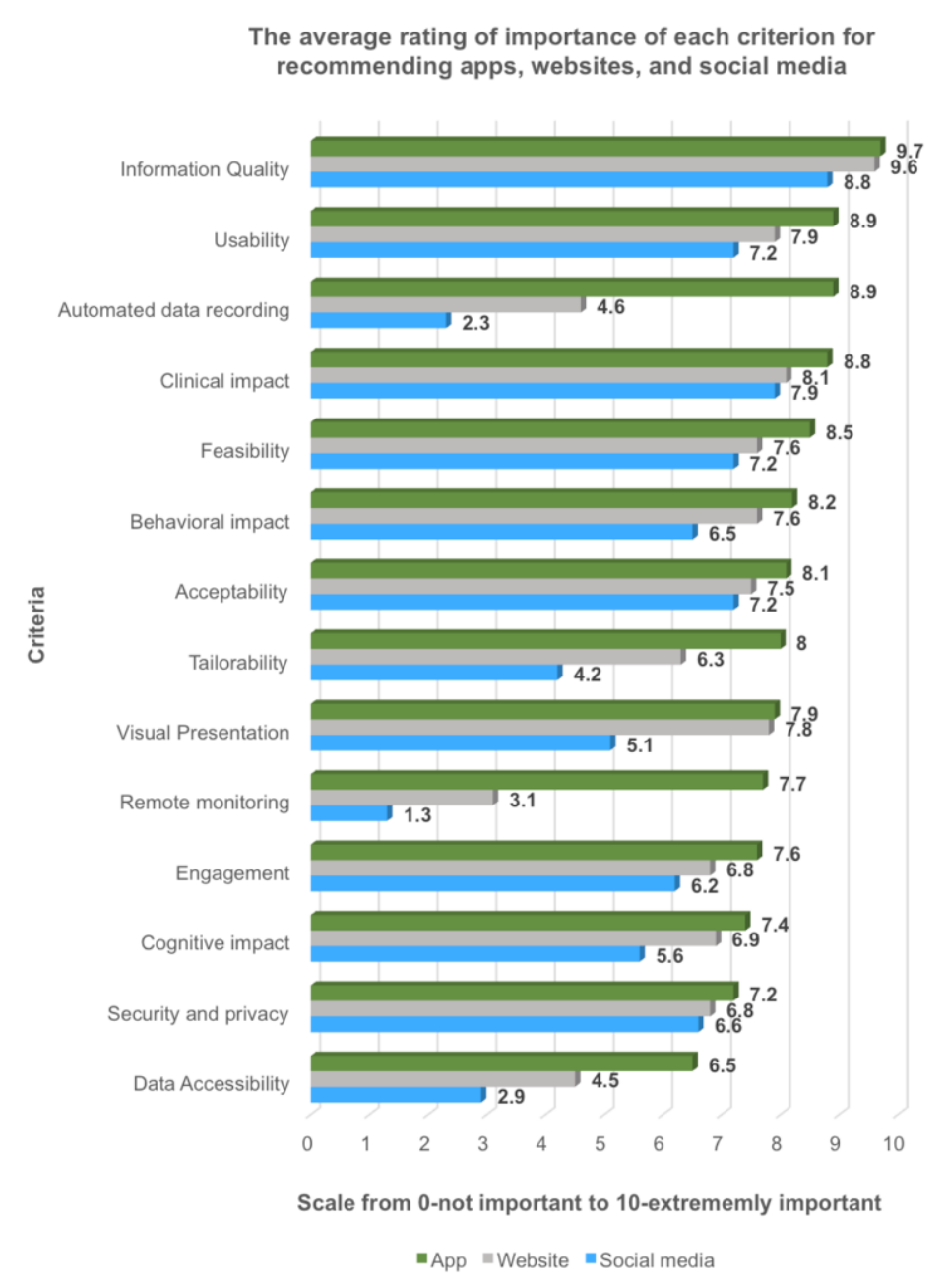

Figure 1. Average rating of importance of the criteria for recommending apps, websites, and social media, given by 15 healthcare professionals in a 3-round Delphi Study.

For assessing digital diabetes self-management tools, Usability was the highest rated criterion for apps, websites, and social media with an average rating of 9.6, 8.6 and 7.4, respectively. The second highest rated criterion was Feasibility with an average rating of 8.6, 7.3, and 7.0, respectively. The third highest rated criterion was Behavioral impact (8.1) for apps, Clinical impact (7.1) for websites, and Acceptability (6.8) for social media. 


\section{Discussion}

With this Delphi study, we elicited the opinions of healthcare professionals on the criteria required to assess and recommend digital self-management tools to individuals with diabetes. The highest rated criteria were Usability and Information quality, respectively. For assessing apps, Security and privacy was one of the lowest rated criteria.

In our study, we found that the panellists consider the Usability of digital diabetes self-management tools essential to their adoption and use. Usability was amongst the three highest rated criteria for both assessing and recommending apps, websites, and social media. Our findings are supported by studies that consider Usability an important criterion in the evaluation and use of developed digital diabetes health tools [9-11]. For recommending diabetes apps, websites, and social media to patients, the panellists unanimously agreed that the quality of the self-management and diabetes-related information (Information quality) was highly important. This is supported by FernandezLlatas [12], who notes that diabetes-related information should be precise and correct to encourage successful self-management.

To be effective as digital self-management tools, apps, websites, and social media should positively impact the health and self-management behaviours of individuals with diabetes [13-15]. The panellists rated Clinical impact and Behavioral impact as equally important for assessing and recommending digital diabetes self-management tools. Feasibility and Acceptability are criteria to consider when assessing and recommending digital self-management tools, especially social media, as the use of this for selfmanagement is still novel and unfamiliar [13] to many individuals with diabetes and their healthcare providers.

Similar to previous findings [7], Security and privacy received little attention as an evaluation criterion. Most of the healthcare professionals assumed that a commercially available app should have fulfilled all necessary security and privacy regulations.

Limitations: Though the panellists' in this study were healthcare professionals with diabetes care experience, they might not represent the perspectives of the average healthcare person supporting patients in the use of digital diabetes tools. Panellists were asked to refer to a specific list of criteria to assess digital diabetes self-management tools targeting the Norwegian population, therefore, the consensus may not apply to other countries or health conditions. Future research is required to explore the opinions of individuals with diabetes and their relatives on the importance of these and other criteria, for this rapidly growing field.

\section{Conclusions}

Digital diabetes tools like apps, sensors, websites, and social media are being increasingly used by patients and healthcare professionals. It is however, challenging for them to assess, choose or recommend the right self-management tools for optimum health benefits for each patient.

Having access to a list of criteria for assessing and recommending these digital diabetes tools could help both patients and healthcare professionals make informed choices in recommending and choosing suitable apps, websites, and social media for self-management. Future work on quality assessment of digital health tools should place emphasis on security and privacy compliance, to enable patients and healthcare professionals focus on other relevant criteria to recommend or choose such tools. 


\section{Acknowledgments}

This project is funded by the Northern Norway Regional Health Authority, Helse Nord (HNF1425-18). We would like to thank the members in our project's Advisory board: Prof. Gunnar Hartvigsen, Dr. Anne Grethe Olsen and Dr. Med. Anne Helen Hansen. We are sincerely grateful to all the healthcare professionals who participated in the study.

\section{References}

[1] Carpenter R, DiChiacchio T, Barker K. Interventions for self-management of type 2 diabetes: An integrative review. Int J Nurs Sci. 2018;6(1):70-91.

[2] Chrvala CA, Sherr D, Lipman RD. Diabetes self-management education for adults with type 2 diabetes mellitus: A systematic review of the effect on glycemic control. Patient Educ Couns. 2016;99(6):926-43.

[3] Kaufman N. Digital Therapeutics: Leading the Way to Improved Outcomes for People With Diabetes. Diabetes Spectr. 2019;32(4):301-3.

[4] Tighe SA, Ball K, Kensing F, Kayser L, Rawstorn JC, Maddison R. Toward a Digital Platform for the Self-Management of Noncommunicable Disease: Systematic Review of Platform-Like Interventions. J Med Internet Res. 2020;22(10):e16774.

[5] Klonoff DC, Kerr D. Overcoming Barriers to Adoption of Digital Health Tools for Diabetes. J Diabetes Sci Technol. 2018;12(1):3-6.

[6] Fink A, Kosecoff J, Chassin M, Brook RH. Consensus methods: characteristics and guidelines for use. Am J Public Health. 1984;74(9):979-83.

[7] Larbi D, Randine P, Årsand E, Antypas K, Bradway M, Gabarron E. Methods and Evaluation Criteria for Apps and Digital Interventions for Diabetes Self-Management: Systematic Review. J Med Internet Res. 2020;22(7):e18480.

[8] Larbi D, Antypas K, Bradway M, Randine P, Gabarron E, Årsand E. How To Choose Among The Many Diabetes Apps And Online Resources? Diabetes Technol Ther. 2020;22(S1):A-229.

[9] Abidi S, Vallis M, Piccinini-Vallis H, Imran SA, Abidi SSR. Diabetes-Related Behavior Change Knowledge Transfer to Primary Care Practitioners and Patients: Implementation and Evaluation of a Digital Health Platform. JMIR Med Inform. 2018;6(2):e25.

[10] Eze ND, Mateus C, Cravo Oliveira Hashiguchi T. Telemedicine in the OECD: An umbrella review of clinical and cost-effectiveness, patient experience and implementation. PLOS ONE. 2020;15(8):e0237585.

[11] Torbjørnsen A, Ribu L, Rønnevig M, Grøttland A, Helseth S. Users' acceptability of a mobile application for persons with type 2 diabetes: a qualitative study. BMC Health Serv Res. 2019;19(1):641.

[12] Fernandez-Llatas C, Traver V, Borras-Morell J-E, Martinez-Millana A, Karlsen R. Are Health Videos from Hospitals, Health Organizations, and Active Users Available to Health Consumers? An Analysis of Diabetes Health Video Ranking in YouTube. Comput Math Methods Med. 2017;8194940.

[13] Elnaggar A, Ta Park V, Lee SJ, Bender M, Siegmund LA, Park LG. Patients' Use of Social Media for Diabetes Self-Care: Systematic Review. J. Med. Internet Res. 2020;22(4):e14209.

[14] Veazie S, Winchell K, Gilbert J, Paynter R, Ivlev I, Eden KB, et al. Rapid Evidence Review of Mobile Applications for Self-management of Diabetes. J Gen Intern Med. 2018;33(7):1167-76.

[15] Yu CH, Parsons J, Mamdani M, Lebovic G, Shah BR, Bhattacharyya O, et al. Designing and evaluating a web-based self-management site for patients with type 2 diabetes - systematic website development and study protocol. BMC Med Inform Decis Mak. 2012;12:57. 